\title{
A Statistical Approach To Texture Description of Medical Images: A Preliminary Study
}

\author{
Matjaž Bevk, Igor Kononenko \\ University of Ljubljana, Faculty of Computer and Information Science, \\ Tržaška 25, SI-1001 Ljubljana, Slovenia, \\ e-mail: \{matjaz.bevk;igor.kononenko\}@fri.uni-lj.si
}

January 29, 2002

\begin{abstract}
The article deals with the problem of texture description. It presents a statistical approach. Specifically it introduces the use of first- and second-order statistics on texture color spaces. At the end we also give estimations of computational time complexities of calculations of parameters presented in this article and describe our experience on one application domain. This study is a preliminary preparation for application of these methods on medical images.
\end{abstract}

\section{Introduction}

Texture is a very commonly used term in computer vision. We all recognize texture when we see it, but it is very difficult to define it precisely. We can find many texture definitions in literature. Coggins[1] for example collected a catalogue of texture definitions found in computer vision literature. Briefly we can regard an image texture as a function of spatial variation in pixel values.

The above is a computer vision viewpoint of texture perception, but let us take a quick look at psychophysics viewpoint of the matter. The question is "When is a texture pair discriminable (by human), given that they have the same brightness, contrast and color?" Julesz $[2,3,4]$ has studied human texture perception in the context of its discrimination. His work concentrates on the spatial statistics of texture gray levels. To summarize Julesz's work, we need to define the first- and second-order spatial statistics.

First-order statistics are calculated from the probability of observing a particular pixel value at a randomly chosen location in the image. They depend only on individual pixel values and not on interaction of neighboring pixel values. The mean of image gray intensity is an example of the first-order statistic.

Second-order statistics are calculated from the probability of observing a pair of pixel values in the image that are some vector $\vec{d}$ apart. Note that second-order statistics become first-order if $\vec{d}=(0,0)$. 
Julesz states that two textures are not preattentively (at first sight) discriminable by human, if their second order statistics are identical. It also means that identity of firstorder statistics can not assure us, that those two textures won't be discriminable by human.

Therefore, if we are to make a system that discriminates textures at least as good as human does, we should take into account both first- and second-order statistics.

The paper is organized as follows. In the second section we define histogram and first order statistics on histogram function. In the third section we define cooccurrence function and second order statistics on cooccurrence function. The fourth section comprehends computational time complexity estimations of functions described in second and third section. In the fifth section we demonstrate the usage of solutions presented in this article on one application domain. The last section is a conclusion with some ideas for improvements and further work.

\section{$2 \quad$ First-order statistics}

First-order statistics are quite straightforward. They are computed from a function that measures the probability of a certain pixel occurring in an image. This function is also known as histogram.

Histogram on gray scale images is defined as follows

$$
H(g)=\frac{n_{g}}{N} ; g=0,1, \ldots, G-1
$$

where $\mathrm{N}$ is the number of all pixels in an image, $\mathrm{G}$ is the number of gray levels and $n_{g}$ is the number of pixels of value $g$ in an image.

What about color images? Let's suppose we use common RGB color space representation. In that case we define histogram on color images like this

$$
\chi(\vec{c})=\frac{n_{\vec{c}}}{N} ; \vec{c} \in \mathcal{L}
$$

where $\mathrm{N}$ is the number of all pixels in an image, $\mathcal{L}$ denotes a three dimensional color space $L \times L \times L$ where $\mathrm{L}$ is the number of intensity levels per color channel and $n_{\vec{c}}$ is the number of pixels of value $\vec{c}$ in an image.

As we can see $\mathrm{H}$ and $\chi$ are probability functions of pixel values, therefore we can characterize their properties with a set of statistical parameters (also called first-order statistics). Below is a list of such parameters, where $P_{n}$ represents $n$-th parameter of $H$ and $\Pi_{m}$ denotes m-th parameter of $\chi$. Note that some $\Pi_{m}$ are vectors from $\mathcal{L}$. For example mean (average color) of an image must also be a color, therefore a vector. But on the other hand some $\Pi_{m}$ are scalars. For example standard deviation is a measure of deviation in the data, therefore not a vector. This is also obvious arithmetically, if you just calculate the parameters.

Mean (average brightness / color)

$$
P_{1}=\sum_{g=0}^{G-1} g H(g) \quad \vec{\Pi}_{1}=\sum_{\vec{c} \in \mathcal{L}} \vec{c} \chi(\vec{c})
$$

\section{Standard deviation (image contrast)}




$$
P_{2}=\sqrt{\sum_{g=0}^{G-1}\left(g-P_{1}\right)^{2} H(g)} \quad \Pi_{2}=\sqrt{\sum_{\vec{c} \in \mathcal{L}}\left(\vec{c}-\vec{\Pi}_{1}\right)^{2} \chi(\vec{c})}
$$

Skew

$$
P_{3}=\frac{\sum_{g=0}^{G-1}\left(g-P_{1}\right)^{3} H(g)}{P_{2}{ }^{3}} \quad \vec{\Pi}_{3}=\frac{\sum_{\vec{c} \in \mathcal{L}}\left(\vec{c}-\vec{\Pi}_{1}\right)^{3} \chi(\vec{c})}{\Pi_{2}{ }^{3}}
$$

Energy

$$
P_{4}=\sum_{g=0}^{G-1} H^{2}(g) \quad \Pi_{4}=\sum_{\vec{c} \in \mathcal{L}} \chi^{2}(\vec{c})
$$

Entropy

$$
P_{5}=-\sum_{g=0}^{G-1} H(g) \log _{2}(H(g)) \quad \Pi_{5}=-\sum_{\vec{c} \in \mathcal{L}} \chi(\vec{c}) \log _{2}(\chi(\vec{c}))
$$

\section{1st moment}

It is the same parameter as mean $P_{6}=P_{1}$ and $\vec{\Pi}_{6}=\vec{\Pi}_{1}$.

\section{2nd moment}

$$
P_{7}=\sum_{g=0}^{G-1}\left(g-P_{6}\right)^{2} H(g) \quad \Pi_{7}=\sum_{\vec{c} \in L \times L \times L}\left(\vec{c}-\vec{\Pi}_{6}\right)^{2} \chi(\vec{c})
$$

3rd moment

$$
P_{8}=\sum_{g=0}^{G-1}\left(g-P_{6}\right)^{3} H(g) \quad \vec{\Pi}_{8}=\sum_{\vec{c} \in \mathcal{L}}\left(\vec{c}-\vec{\Pi}_{6}\right)^{3} \chi(\vec{c})
$$

4th moment

$$
P_{9}=\sum_{g=0}^{G-1}\left(g-P_{6}\right)^{4} H(g) \quad \Pi_{9}=\sum_{\vec{c} \in \mathcal{L}}\left(\vec{c}-\vec{\Pi}_{6}\right)^{4} \chi(\vec{c})
$$

\section{Second-order statistics}

As it was already mentioned above, second-order statistics operate on probability function, that measures the probability of a pair of pixel values occurring some vector $\vec{d}$ apart in the image. This probability function is also called cooccurrence matrix, since it measures the probability of cooccurrence of two pixel values. Since cooccurrence matrix becomes "weird" in color space, let us use the term cooccurrence function (CF) instead.

Let us now define $\mathrm{CF}$ on the gray scale image. Suppose we have a $M \times N$ image with color space of $\mathrm{G}$ gray levels. Given a certain displacement vector $+\vec{d}=(d x, d y)$ the $\mathrm{CF}$ looks like this

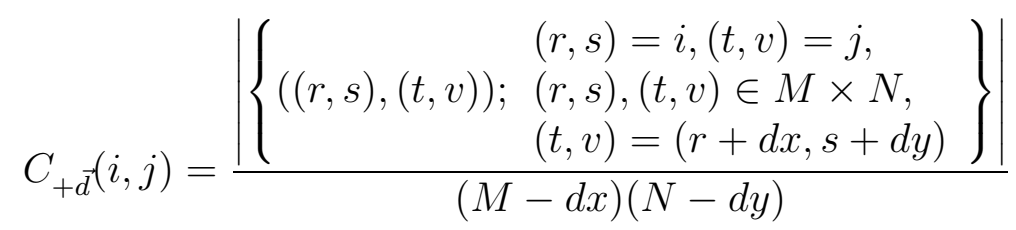


Note that so defined $\mathrm{CF}$ is not symmetrical in terms of $C_{+\vec{d}}(i, j) \neq C_{+\vec{d}}(j, i)$. A symmetrical $\mathrm{CF}$ can be computed using the formula

$$
C_{\vec{d}}(i, j)=C_{+\vec{d}}(i, j)+C_{-\vec{d}}(i, j)
$$

Suppose we have a set of displacement vectors $\Delta=\left\{\vec{d}_{1}, \vec{d}_{2}, \ldots, \vec{d}_{n}\right\}$. The final CF $C$ used in second-order statistics is then the average at certain gray level $(i, j)$ over all CFs based on displacement vectors from $\Delta$.

$$
C(i, j)=\operatorname{avg}\left\{C_{\vec{d}_{k}}(i, j) ; \vec{d}_{k} \in \Delta\right\}
$$

CFs on color images can be defined in a similar way. Suppose we have a $M \times N$ image with an ordinary three dimensional RGB color space. Let's say that each color channel has $L$ values. Therefore each image pixel is a vector from $\mathcal{L}$ which is a $L \times L \times L$ space. Given a certain displacement vector $+\vec{d}=(d x, d y)$ we get the $\mathrm{CF}$

$$
\Gamma_{+\vec{d}}\left(\vec{c}_{1}, \vec{c}_{2}\right)=\frac{\left|\left\{\begin{array}{c}
(r, s)=\vec{c}_{1},(t, v)=\vec{c}_{2}, \\
((r, s),(t, v)) ; \\
(r, s),(t, v) \in M \times N, \\
(t, v)=(r+d x, s+d y)
\end{array}\right\}\right|}{(M-d x)(N-d y)}
$$

Notice the difference: $\Gamma_{+\vec{d}}$ is a function of two three dimensional vectors (two RGB colors). And again we can define a symmetrical $\Gamma_{\vec{d}}$

$$
\Gamma_{\vec{d}}\left(\vec{c}_{1}, \vec{c}_{2}\right)=\Gamma_{+\vec{d}}\left(\vec{c}_{1}, \vec{c}_{2}\right)+\Gamma_{-\vec{d}}\left(\vec{c}_{1}, \vec{c}_{2}\right)
$$

The final CF $\Gamma$ used in second-order statistics on color images is the average at certain RGB color pair $\left(\vec{c}_{1}, \vec{c}_{2}\right)$ over all CFs based on displacement vectors from $\Delta$.

$$
\Gamma\left(\vec{c}_{1}, \vec{c}_{2}\right)=\operatorname{avg}\left\{\Gamma_{\vec{d}_{k}}\left(\vec{c}_{1}, \vec{c}_{2}\right) ; \vec{d}_{k} \in \Delta\right\}
$$

The next important issue, which is important on color as well as on gray scale images, is: Exactly which displacement vectors $\vec{d}$ are in $\Delta$ ? There's no definite answer, but there are some clues that came from practice. Mostly used set of distance vectors is $\Delta=\{(1,0),(1,1),(0,1),(-1,1)\}$. This actually means, that we are considering four neighboring pixels. It is empirically proven that this is a rather successful set, but there is no proof that this is a universally good set. This set holds pretty good information on local (short distance) pixel dependancies, but lacks of global (long distance) pixel dependancies. The more we extend $\Delta$ with vectors that represent larger distances, the more local information we loose. This loss of information happens because of the averaging in the last step of calculation of CF. There is obviously a trade off between local and global information that $\mathrm{CF}$ could hold.

In order to describe CF $C$ of gray scale image Haralick[5] proposed a set of secondorder statistics. These statistics and their generalized versions (statistics for $\Gamma$ ) are listed below in this article. In the list below $F_{n}$ represents n-th statistics of $C$ and $\Phi_{m}$ denotes m-th statistic of $\Gamma$. 


\section{Energy}

$$
F_{1}=\sum_{i=0}^{G-1} \sum_{j=0}^{G-1} C^{2}(i, j) \quad \Phi_{1}=\sum_{\vec{c}_{1} \in \mathcal{L}} \sum_{\overrightarrow{c_{2}} \in \mathcal{L}} \Gamma^{2}\left(\vec{c}_{1}, \vec{c}_{2}\right)
$$

\section{Entropy}

$$
\begin{gathered}
F_{2}=-\sum_{i=0}^{G-1} \sum_{j=0}^{G-1} C(i, j) \log _{2}(C(i, j)) \\
\Phi_{2}=-\sum_{\overrightarrow{c_{1}} \in \mathcal{L}} \sum_{\overrightarrow{c_{2}} \in \mathcal{L}} \Gamma\left(\vec{c}_{1}, \vec{c}_{2}\right) \log _{2}\left(\Gamma\left(\vec{c}_{1}, \vec{c}_{2}\right)\right)
\end{gathered}
$$

\section{Contrast}

$$
F_{3}=\sum_{i=0}^{G-1} \sum_{j=0}^{G-1}(i-j)^{2} C(i, j) \quad \Phi_{3}=\sum_{\overrightarrow{c_{1}} \in \mathcal{L}} \sum_{\overrightarrow{c_{2}} \in \mathcal{L}}\left(\vec{c}_{1}-\vec{c}_{2}\right)^{2} \Gamma\left(\vec{c}_{1}, \vec{c}_{2}\right)
$$

\section{Homogeneity}

$$
F_{4}=\sum_{i=0}^{G-1} \sum_{j=0}^{G-1} \frac{C(i, j)}{1+(i-j)^{2}} \quad \Phi_{4}=\sum_{\overrightarrow{c_{1}} \in \mathcal{L}} \sum_{\vec{c}_{2} \in \mathcal{L}} \frac{\Gamma\left(\vec{c}_{1}, \vec{c}_{2}\right)}{1+\left(\vec{c}_{1}-\vec{c}_{2}\right)^{2}}
$$

\section{Correlation of $C$}

Let us first define marginal probabilities of $C$

$$
C^{\prime}(i)=\sum_{j=0}^{G-1} C(i, j) \quad C^{\prime \prime}(j)=\sum_{i=0}^{G-1} C(i, j)
$$

$C^{\prime}=C^{\prime \prime}$ since $C$ is symmetrical. Notice what is happening here: A probability of a certain $C^{\prime}(i)$ is calculated so that probabilities over all possible pairs of $i$ are summed. What we get is a probability of pixel value $i$ itself. That is actually a histogram of an image. The same holds for $C^{\prime \prime}$ as well.

And let $\mu_{C^{\prime}}$ and $\mu_{C^{\prime \prime}}$ be the means of marginal probabilities

$$
\mu_{C^{\prime}}=\sum_{i=0}^{G-1} i C^{\prime}(i) \quad \mu_{C^{\prime \prime}}=\sum_{j=0}^{G-1} j C^{\prime \prime}(j)
$$

Note that $\mu_{C^{\prime}}=\mu_{C^{\prime \prime}}$. If $C^{\prime}$ and $C^{\prime \prime}$ are histograms of an image and $\mu_{C^{\prime}}$ and $\mu_{C^{\prime \prime}}$ are their means, we can easily see what $\mu_{C^{\prime}}$ and $\mu_{C^{\prime \prime}}$ represent. They simply represent the average pixel value of an image.

Further let $\sigma_{C^{\prime}}$ and $\sigma_{C^{\prime \prime}}$ be the standard deviations of $C^{\prime}$ and $C^{\prime \prime}$

$$
\sigma_{C^{\prime}}=\sqrt{\sum_{i=0}^{G-1}\left(i-\mu_{C^{\prime}}\right)^{2} C^{\prime}(i)} \quad \sigma_{C^{\prime \prime}}=\sqrt{\sum_{j=0}^{G-1}\left(j-\mu_{C^{\prime \prime}}\right)^{2} C^{\prime \prime}(j)}
$$

Obviously $\sigma_{C^{\prime}}=\sigma_{C^{\prime \prime}}$. Now we are ready to define a measure of correlation for $C$, that is

$$
F_{5}=\frac{\sum_{i=0}^{G-1} \sum_{j=0}^{G-1}\left(i-\mu_{C^{\prime}}\right)\left(j-\mu_{C^{\prime \prime}}\right) C(i, j)}{\sigma_{C^{\prime}} \sigma_{C^{\prime \prime}}}
$$




\section{Correlation of $\Gamma$}

Let us first define marginal probabilities of $\Gamma$

$$
\Gamma^{\prime}\left(\vec{c}_{1}\right)=\sum_{\overrightarrow{c_{2}} \in \mathcal{L}} \Gamma\left(\vec{c}_{1}, \vec{c}_{2}\right) \quad \Gamma^{\prime \prime}\left(\vec{c}_{2}\right)=\sum_{\vec{c}_{1} \in \mathcal{L}} \Gamma\left(\vec{c}_{1}, \vec{c}_{2}\right)
$$

$\Gamma^{\prime}=\Gamma^{\prime \prime}$ since $\Gamma$ is symmetrical. $\Gamma^{\prime}$ and Gamma $a^{\prime \prime}$ are also histograms of an image, as we explained it before in gray scale case.

And let $\mu_{\Gamma^{\prime}}$ and $\mu_{\Gamma^{\prime \prime}}$ be the means of marginal probabilities

$$
\vec{\mu}_{\Gamma^{\prime}}=\sum_{\vec{c}_{1} \in \mathcal{L}} \vec{c}_{1} \Gamma^{\prime}\left(\vec{c}_{1}\right) \quad \vec{\mu}_{\Gamma^{\prime \prime}}=\sum_{\overrightarrow{c_{2}} \in \mathcal{L}} \vec{c}_{2} \Gamma^{\prime \prime}\left(\vec{c}_{2}\right)
$$

Note that now $\vec{\mu}_{\Gamma^{\prime}}$ and $\vec{\mu}_{\Gamma^{\prime \prime}}$ are vectors (remember they represent average colors) and it still holds that $\vec{\mu}_{\Gamma^{\prime}}=\vec{\mu}_{\Gamma^{\prime \prime}}$.

Further let $\sigma_{\Gamma^{\prime}}$ and $\sigma_{\Gamma^{\prime \prime}}$ be the standard deviations of $\Gamma^{\prime}$ and $\Gamma^{\prime \prime}$

$$
\sigma_{\Gamma^{\prime}}=\sqrt{\sum_{\vec{c}_{1} \in \mathcal{L}}\left(\vec{c}_{1}-\vec{\mu}_{\Gamma^{\prime}}\right)^{2} \Gamma^{\prime}\left(\vec{c}_{1}\right)} \quad \sigma_{\Gamma^{\prime \prime}}=\sqrt{\sum_{\vec{c}_{2} \in \mathcal{L}}\left(\vec{c}_{2}-\vec{\mu}_{\Gamma^{\prime \prime}}\right)^{2} \Gamma^{\prime \prime}\left(\vec{c}_{2}\right)}
$$

Obviously $\sigma_{\Gamma^{\prime}}=\sigma_{\Gamma^{\prime \prime}}$. And finally we are able to define a measure of correlation for $\Gamma$, that is

$$
\Phi_{5}=\frac{\sum_{\vec{c}_{1} \in \mathcal{L}} \sum_{\vec{c}_{2} \in \mathcal{L}}\left(\vec{c}_{1}-\vec{\mu}_{\Gamma^{\prime}}\right)\left(\vec{c}_{2}-\vec{\mu}_{\Gamma^{\prime \prime}}\right) \Gamma\left(\vec{c}_{1}, \vec{c}_{2}\right)}{\sigma_{\Gamma^{\prime}} \sigma_{\Gamma^{\prime \prime}}}
$$

\section{Variance}

$$
F_{6}=\sum_{i=0}^{G-1} \sum_{j=0}^{G-1}\left(i-\mu_{C^{\prime}}\right)^{2} C(i, j) \quad \Phi_{6}=\sum_{\overrightarrow{c_{1}} \in \mathcal{L}} \sum_{\vec{c}_{2} \in \mathcal{L}}\left(\vec{c}_{1}-\vec{\mu}_{\Gamma^{\prime}}\right)^{2} \Gamma\left(\vec{c}_{1}, \vec{c}_{2}\right)
$$

\section{Mean of pair-sums}

First define a new probability function. A function that represents a probability of a particular pair-sum of pixel values. Let's denote it with $S$ in gray scale and $\Theta$ in color space.

$$
S(x)=\sum_{i+j=x} C(i, j) \quad \Theta(\vec{y})=\sum_{\vec{c}_{1}+\vec{c}_{2}=\vec{y}} \Gamma\left(\vec{c}_{1}, \vec{c}_{2}\right)
$$

Now we can calculate their means

$$
F_{7}=\sum_{x=0}^{2(G-1)} x S(x) \quad \vec{\Phi}_{7}=\sum_{\vec{y} \in \widehat{\mathcal{L}}} \vec{y} \Theta(\vec{y})
$$

where $\widehat{\mathcal{L}}$ is a $2 L \times 2 L \times 2 L$ space.

\section{Variance of pair-sums}

$$
F_{8}=\sum_{x=0}^{2(G-1)}\left(x-F_{7}\right)^{2} S(x) \quad \Phi_{8}=\sum_{\vec{y} \in \widehat{\mathcal{L}}}\left(\vec{y}-\vec{\Phi}_{7}\right)^{2} \Theta(\vec{y})
$$

\section{Entropy of pair-sums}

$$
F_{9}=-\sum_{x=0}^{2(G-1)} S(x) \log (S(x)) \quad \Phi_{9}=-\sum_{\vec{y} \in \widehat{\mathcal{L}}} \Theta(\vec{y}) \log _{2} \Theta(\vec{y})
$$




\section{Variance of pair-differences}

Like we defined a probability function for pair-sums we can also define a probability function for all possible pair-differences. We will denote it with $D$ in gray scale and $\Lambda$ in color space.

$$
D(x)=\sum_{|i-j|=x} C(i, j) \quad \Lambda(\vec{y})=\sum_{\begin{array}{l}
\left|r_{1}-r_{2}\right|=r \\
\left|g_{1}-g_{2}\right|=g \\
\left|b_{1}-b_{2}\right|=b
\end{array}} \Gamma\left(\vec{c}_{1}, \vec{c}_{2}\right)
$$

where $\vec{c}_{1}=\left(r_{1}, g_{1}, b_{1}\right), \vec{c}_{2}=\left(r_{2}, g_{2}, b_{2}\right)$ and $\vec{y}=(r, g, b)$. Now it is possible to define both means of pair-differences

$$
\mu_{D}=\sum_{x=0}^{G-1} x D(x) \quad \vec{\mu}_{\Lambda}=\sum_{\vec{y} \in \mathcal{L}} \vec{y} \Lambda(\vec{y})
$$

And finally we are able to calculate variances

$$
F_{10}=\sum_{x=0}^{G-1}\left(x-\mu_{D}\right)^{2} D(x) \quad \Phi_{10}=\sum_{\vec{y} \in \mathcal{L}}\left(\vec{y}-\vec{\mu}_{\Lambda}\right)^{2} \Lambda(\vec{y})
$$

\section{Entropy of pair-differences}

$$
F_{11}=-\sum_{x=0}^{G-1} D(x) \log (D(x)) \quad \Phi_{11}=-\sum_{\vec{y} \in \mathcal{L}} \Lambda(\vec{y}) \log _{2} \Lambda(\vec{y})
$$

\section{Information measure of correlation 1}

$$
\begin{gathered}
F_{12}=\frac{F_{2}+\sum_{i=0}^{G-1} \sum_{j=0}^{G-1} C(i, j) \log _{2}\left(C^{\prime}(i) C^{\prime \prime}(j)\right)}{-\sum_{i=0}^{G-1} C^{\prime}(i) \log _{2}\left(C^{\prime}(i)\right)} \\
\Phi_{12}=\frac{\Phi_{2}+\sum_{\vec{c}_{1} \in \mathcal{L}} \sum_{\vec{c}_{2} \in \mathcal{L}} \Gamma\left(\vec{c}_{1}, \vec{c}_{2}\right) \log _{2}\left(\Gamma^{\prime}\left(\vec{c}_{1}\right) \Gamma^{\prime \prime}\left(\vec{c}_{2}\right)\right)}{-\sum_{\vec{c}_{1} \in \mathcal{L}} \Gamma^{\prime}\left(\vec{c}_{1}\right) \log _{2}\left(\Gamma^{\prime}\left(\vec{c}_{1}\right)\right)}
\end{gathered}
$$

\section{Information measure of correlation 2}

$$
\begin{gathered}
F_{13}=\sqrt{\left|1-e^{-2\left(-\sum_{i=0}^{G-1} \sum_{j=0}^{G-1} C^{\prime}(i) C^{\prime \prime}(j) \log _{2}\left(C^{\prime}(i) C^{\prime \prime}(j)\right)+F_{2}\right)}\right|} \\
\Phi_{13}=\sqrt{\left|1-e^{-2\left(-\sum_{\vec{c}_{1} \in \mathcal{L}} \sum_{\vec{c}_{2} \in \mathcal{L}} \Gamma^{\prime}\left(\vec{c}_{1}\right) \Gamma^{\prime \prime}\left(\vec{c}_{2}\right) \log _{2}\left(\Gamma^{\prime}\left(\vec{c}_{1}\right) \Gamma^{\prime \prime}\left(\vec{c}_{2}\right)\right)+\Phi_{2}\right)}\right|}
\end{gathered}
$$

\section{Time Complexity}

It is important to analyse computational time complexity of these functions. As they may be simply written in math formulae they tend to be quite algorithmically time and space consuming.

In the following subsections we will use: $M$ and $N$ to denote a $M \times N$ image, $G$ for gray scale channel and $L$ for the size of color channel. 


\subsection{Time Complexity of Histogram function}

What we do here is run over all image pixels and count them. That means we have to do $M \cdot N$ steps. That is usually feasible for all normal image sizes in gray scale and color space.

\subsection{Time Complexity of First-order Statistics}

Note that all first-order statistics require the sum over all points of gray/color space. That is the most time consuming part of them.

In gray scale space

$$
\sum_{g=0}^{G-1} F u n c t i o n \_o f(H(g))
$$

which gives $G$ loops. Considering usual values for $G$ the computational complexity is acceptable with current hardware speed.

In color space we have

$$
\sum_{\vec{c} \in \mathcal{L}} F \text { unction_of }(\chi(\vec{c}))
$$

which requires $L^{3}$ loops. Now imagine, we use usual quantization of a signal per color channel $(L=256)$, then $L^{3}$ is approximately 16.7 million! Therefore, it is recommended to use a rougher quantization, say $L=16$.

\subsection{Time Complexity of CF}

Remember how we calculate CF. First we count all pair pixels that are some vector $+\vec{d}$ apart. We can do that in approximately $M \cdot N$ steps. Note the size of the CF space. It is $G \times G$ for gray scale and $L \times L \times L \times L \times L \times L$ for color images! Then we calculate a symmetrical $\mathrm{CF}$, which requires summing over all gray/color space. That means $G^{2}$ summations in gray scale and $L^{6}$ summations in color space. And lastly we average CFs for all displacement vectors. Which again requires $G^{2}$ and $L^{6}$ operations respectively. Needless to say, it is recommended to use a rougher quantizations than 256 values per channel.

\subsection{Time Complexity of Second-order Statistics}

The most time consuming part of second-order statistics is obviously summing over all points from its space.

In gray scale space this part looks like this

$$
\sum_{i=0}^{G-1} \sum_{j=0}^{G-1} \text { Function_of }(C(i, j))
$$

which requires $G^{2}$ operations.

And in color space we have

$$
\sum_{\overrightarrow{c_{1}} \in \mathcal{L}} \sum_{\vec{c}_{2} \in \mathcal{L}} \text { Function_of }\left(\Gamma\left(\vec{c}_{1}, \vec{c}_{2}\right)\right)
$$

which requires $L^{6}$ operations. 


\section{Application}

In this section we describe one application of the approach presented in previous sections. The task is to discriminate two chemical suspensions on the basis of their images of dried drops.

For our experiment, the first suspension was $0.1 \%$ of $\mathrm{Al}_{2} \mathrm{O}_{3}$ in $\mathrm{H}_{2} \mathrm{O}$ with acidity of $\mathrm{Ph}$ 6, the second suspension was also $0.1 \%$ of $\mathrm{Al}_{2} \mathrm{O}_{3}$ in $\mathrm{H}_{2} \mathrm{O}$ but with acidity of $\mathrm{Ph} 10$.

\section{$5.1 \quad$ Procedure}

The procedure was as follows. First the suspensions were prepared. The second task was to make drop samples of the suspensions. Drop samples were made with a sterile syringe on a clean microscopic slide. 8 drops per slide were made ( 4 from $\mathrm{Ph} 6$ suspension and 4 from $\mathrm{Ph} 10$ suspension). The average diameter of a drop was about $5 \mathrm{~mm}$. At this point the drops were dried before further processing. To take pictures of dried drops, we used a dark field microscope with a mounted digital camera. A relatively small magnification factor of 20 was used. Each drop separately was observed under microscope and photographed (Fig. 1, Fig. 2). 12 slides of drops were sampled and we got 45 images of $\mathrm{Ph} 6$ and 37 images of $\mathrm{Ph} 10$ suspension. Some images had to be eliminated due to inappropriate size of a drop (some drops were too big to fit on the image).

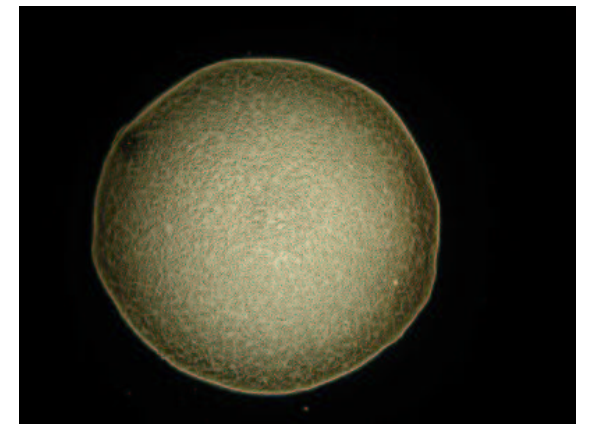

Fig. 1: An image of $\mathrm{Ph} 6$ droplet

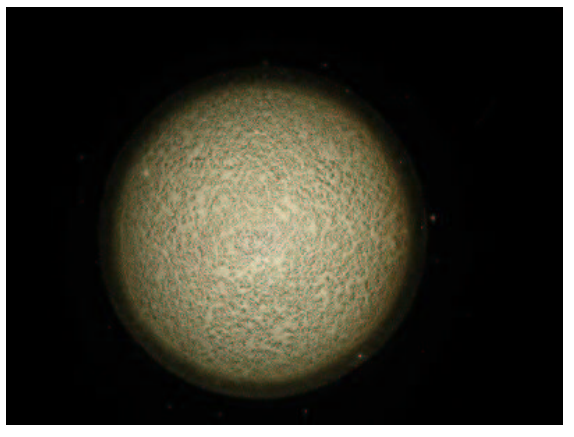

Fig. 2: An image of $\mathrm{Ph} 10$ droplet

The size of images was $2048 \times 1536$. The Color depth was 24 bit.

In the next step we extracted an inner rectangular area of each drop in order to obtain a discriminating pattern (Fig. 3, Fig. 4).

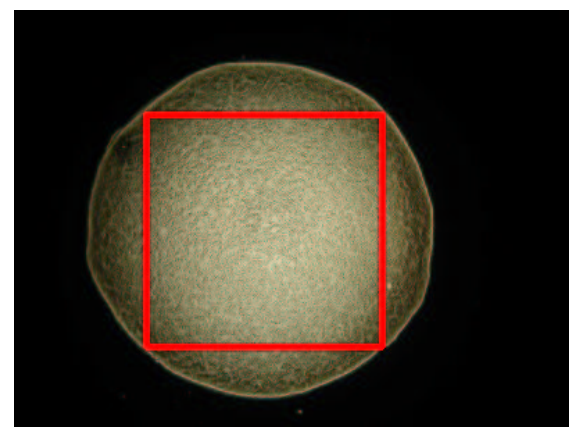

Fig. 3: An area marked for extraction

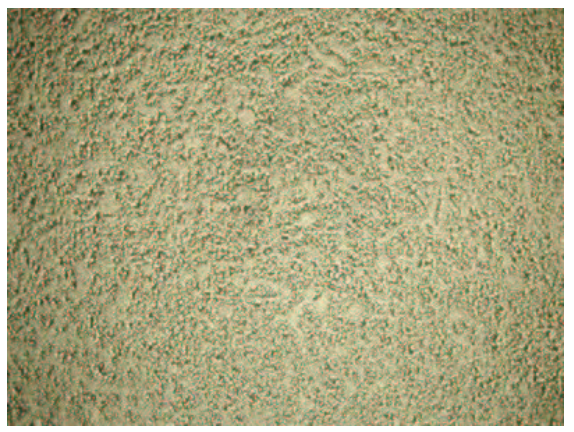

Fig. 4: An extracted area 
So preprocessed images were used in the next phase of the experiment, where we calculated the histogram and CF parameters. The calculation of parameters was performed by our program ImageProcessor (IP), that implements all the parameters described in Sec. 2 and 3. The output of IP was a list of samples (images) described by histogram

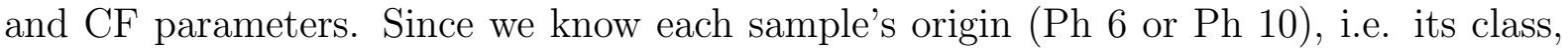
we tried to solve this classification problem in order to verify the quality of parameters.

The task is to classify samples into one of two classes $\mathrm{Ph} 6$ and $\mathrm{Ph} 10$ using histogram and $\mathrm{CF}$ parameters. We had $45+37$ samples, which means the default classification error of 45\%. The aim is to minimize the classification error. We used See5[7] (C5.0 algorithm), that builds decision trees. Each experiment was 10 fold cross validated.

\subsection{Results}

In the first experiment we tried the parameters on gray scale images. Each image was color-quantized to 256 levels for histogram and 16 levels for CF parameters. The classification error on gray scale images was $5.0 \% \pm 2.8 \%$, written in the form of mean \pm standard error (Tab. 1a).

In the second experiment we tried parameters on color images. Each image was colorquantized to 16 levels per channel for histogram and 8 levels per channel for CF parameters. The classification error on color images was $1.3 \% \pm 1.3 \%$ (Tab. 1b).

\begin{tabular}{|c|c|c|c|c|c|}
\hline \multirow{3}{*}{ Fold } & \multicolumn{2}{|c|}{ Decision Tree } & \multirow{3}{*}{ Fold } & \multicolumn{2}{|c|}{ Decision Tree } \\
\hline & --- & ----- & & - & -- \\
\hline & Size & Errors & & Size & Errors \\
\hline 0 & 3.0 & $12.5 \%$ & 0 & 2.0 & $0.0 \%$ \\
\hline 1 & 3.0 & $0.0 \%$ & 1 & 2.0 & $0.0 \%$ \\
\hline 2 & 3.0 & $0.0 \%$ & 2 & 2.0 & $0.0 \%$ \\
\hline 3 & 3.0 & $12.5 \%$ & 3 & 2.0 & $0.0 \%$ \\
\hline 4 & 3.0 & $0.0 \%$ & 4 & 2.0 & $12.5 \%$ \\
\hline 5 & 3.0 & $0.0 \%$ & 5 & 2.0 & $0.0 \%$ \\
\hline 6 & 3.0 & $25.0 \%$ & 6 & 2.0 & $0.0 \%$ \\
\hline 7 & 3.0 & $0.0 \%$ & 7 & 2.0 & $0.0 \%$ \\
\hline 8 & 3.0 & $0.0 \%$ & 8 & 2.0 & $0.0 \%$ \\
\hline 9 & 3.0 & $0.0 \%$ & 9 & 2.0 & $0.0 \%$ \\
\hline Mean & 3.0 & $5.0 \%$ & Mean & 2.0 & $1.3 \%$ \\
\hline $\mathrm{SE}$ & 0.0 & $2.8 \%$ & $\mathrm{SE}$ & 0.0 & $1.3 \%$ \\
\hline
\end{tabular}

Tab. 1: The results of see5 on (a) gray scale images and (b) color images

The results prove the information quality of parameters. The result on color images is better than on gray scale ones, which shows the importance of color in patterns. Notice that, though we used rougher quantization on color images, the result is better than on gray scale images. 


\section{Conclusion}

It is possible to describe a certain texture statistically. The quality of description depends on complexity of statistics, in other words, on computational time and space. Histogram alone may be too simple to give a good description, whereas CFs could do the job. The use of CFs makes sense also because they can compete with humans[2, 3, 4]. Anyway, further work could continue in two directions:

- To find new better second-order statistics.

- To try statistics of higher orders.

It would also be helpful, if we found a computationally less expensive representation of CFs. Here is a potential solution. Generally we have a relatively huge color space with relatively small number of points in it. For example: Say we have a $1024 \times 768(M=$ 1024, $N=768$ ) color image, with 256 values per color channel $(L=256)$. Calculating its $\mathrm{CF}$ would require more than $256^{6} \doteq 2.8 \cdot 10^{14}$ steps. But we know that we have at most $1024 \cdot 768 \doteq 7.8 \cdot 10^{5}$ points in that space. It would be much faster, if we store the relevant points and their values (probabilities) and then scan through points rather than through the whole space.

This is a preliminary study of image analysis. The aim is to use these methods in medical image analysis. For example analysis of pulmonary scintigraphic images. The idea is to learn to discriminate images of healthy from sick lungs.

\section{Acknowledgements}

We thank dr. Saša Novak from Jožef Stefan Institute, for her help with experiments and for preparation of chemical suspensions. We also thank Minnie Hein from University of Stuttgart for helping us with experiments and for providing the experimental equipment. This research was supported by the Slovenian Ministry of Education, Science and Sports. 


\section{References}

[1] Coggins J.M., A Framework for Texture Analysis on Spatial Filtering, Ph.D. Thesis, Computer Science Department, Michigan State University, East Lansing, Michigan, 1982. (cited in [6])

[2] Julesz B., Gilbert E.N., Shepp L.A., Frisch H.L., Inability of Humans To Discriminate Between Visual Textures That Agree in Second-Order-Statistics, Perception 2, pp. 391405, 1973. (cited in [6])

[3] Julesz B., Visual Pattern Discrimination, IRE Trans. on Information Theory, IT-8, pp.84-92, 1962. (cited in [6])

[4] Julesz B., Experiments In The Visual Perception of Texture, Scientific American, 232, pp. 34-43, 1975. (cited in [6])

[5] Haralick R.M., Shanmugam K., Dinstein I., Textural Features for Image Classification, IEEE Transactions on Systems, Man and Cybernetics, pp.610-621, 1973.

[6] Chen C.H., Pau L.F., Wang P.S.P., The Handbook of Pattern Recognition and Computer Vision (2nd Edition), World Scientific Publishing Co., pp.207-248, 1998.

[7] Quinlan J.R., C4.5 programs for machine learning, Morgan Kaufmann, 1993. 